\title{
Study of free convection by infrared thermography over a constant heat-flux heated plate
}

\author{
by VERMEULEN J.P. and BAUDOIN B.
}

Département Energétique Industrielle, Ecole des Mines de Douai

941, Rue Charles Bourseul, BP 838, 59508 Douai Cedex, France.

\begin{abstract}
This study presents experimental free convection heat transfer correlations obtained, in case of air, over vertical and inclined flat plate as well as vertical plate with transverse disturbing elements located in the laminar boundary layer. In the last case, the aim of this study is to define the geometric configuration giving rise to the largest increase of the heat transfer performance. Temperature measurements were performed using IR thermography in parallel with a satisfactory experimental procedure. These measurements were also completed with some flow visualizations.
\end{abstract}

\section{Nomenclature.}

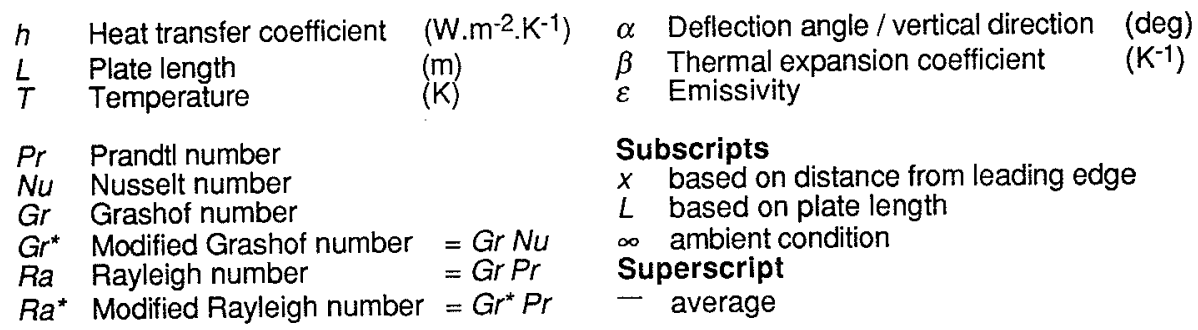

\section{Introduction}

The study of heat transfer by free convection presents a great interest in industrial applications although this kind of heat exchange is relatively low compared to the forced convection phenomenon. From previous investigations, we expect a non dimensional heat transfer law $N u_{x}=A\left(R a^{*}\right)^{0.2}$ for constant heat flux heated plate (where $A$ is a function of Prandtl number). The experimental results obtained in the scope of this study are compared to those obtained in the literature and the future objectives are developed in this article.

\section{Theoretical and experimental results}

\subsection{Theoretical correlations}

For constant heat-flux heated plate in laminar free convection, boundary layer equations can be numerically solved using similarity variables [1-2]. In the case of air $(\operatorname{Pr}=0.7)$, we obtain : $N u_{x}=0.486\left(G r_{x}^{*}\right)^{0.2}$, or

$$
N u_{x}=0.522\left(R a^{*}\right)^{0.2}
$$

The problem of laminar free convection over a constant heat flux heated plate can also be treated using von Kàrman-Polhausen integral relations [3] giving for $\mathrm{Pr}=0.7$ :

$$
N u_{x}=0.529\left(R a^{*}\right)^{0.2}
$$




\subsection{Existing experimental correlations}

DOTSON [4] proposed a similar correlation valid for $\operatorname{Pr}=0.7$ :

$$
N u_{x}=0.55\left(R a_{x}^{*}\right)^{0.2}
$$

CHEN et $A l$. [5] proposed in case of vertical and inclined flat plate (hot surface facing downward) :

$$
N u_{x}=0.532\left(R a^{*}{ }_{x} \cos \alpha\right)^{0.2}
$$

\section{Present experimental study}

\subsection{Model configuration}

The experimental setup used in the scope of this study is presented in figure 1 . It is composed by a table surrounded with lateral windows (except between the IR camera and the plate) in order to prevent outside airstream capable of interfering with the free convection phenomenon. In this box, the flat plate is fixed with an adjustable support allowing different angles of inclination. The IR camera is set in front of the plate, perpendicular to it. This plate, described in more detail in figure 2 , is composed by a flat electric resistance $(0.1 \mathrm{~m} \times 0.2 \mathrm{~m})$ inserted between a thermal insulator $(3 \mathrm{~cm}$ thick) and a glass plate $(3 \mathrm{~mm}$ thick) painted with an high emissivity paint $(\varepsilon \cong 0.93$ in the spectral range of the camera). Between the thermal insulator and the resistance, three thermocouples are placed in order to measure the surface temperature of the insulator (which is limited to $80^{\circ} \mathrm{C}$ ) and to know when the thermal steady state is reached. The electric resistance is set so that the thermal and dynamic boundary layers have the same origin (for more details, see [6]).

\subsection{Experimental study}

\subsubsection{Introduction}

An Agema model 870 infrared camera was used for this study. It is equipped with a thermoelectrically cooled $\mathrm{HgCdTe}$ detector sensitive in the $2-5 \mu \mathrm{m}$ band. The field of view is scanned by two mirrors; the horizontal rotating mirror allows a scanning at $2500 \mathrm{~Hz}$, the vertical oscillating at $25 \mathrm{~Hz}$. Each field is composed by 70 useful horizontal lines or 280 lines per frames (4:1 interlacing). The thermal resolution is given to be $0.1^{\circ} \mathrm{C}$.

\subsubsection{IR camera calibration}

In order to convert the isotherm units, delivered by the camera, into temperatures, a calibration is necessary. This is done using a $10 \mathrm{~cm} \times 10 \mathrm{~cm}$ highly conductive plate (painted with the same paint as the model) on which a small dimensions thermocouple is glued. This plate is heated via an electric resistance connected to an adjustable constant current source allowing a quasi steady state calibration. The resulting calibration curve is presented in figure 3 and shows a strong non linear response. Figure 4 presents the associated uncertainty range of the IR camera calibration allowing temperature measurement with an accuracy of $\pm 0.2^{\circ} \mathrm{C}$.

\subsubsection{Data acquisition and reduction}

The infrared signal delivered by the camera is directly stored in real time on a PC by means of the PTR 9000 data acquisition software developed by CEDIP I3S. This software reconstructs fields composed of 64 lines of 128 or 256 points per line with a 12-bit accuracy (4096 thermal levels).

After the conversion of the isotherm units into temperatures, the data reduction program evaluates $N u_{x}$ and $\mathrm{Ra}^{*}{ }_{x}$ (the fluid properties are evaluated at the film temperature except $\beta$ which is calculated using $T_{\infty}$ ). Due to the large temperature difference between the plate and the ambient atmosphere (of the order of $50^{\circ} \mathrm{C}$ ), the convective heat flux is calculated from the electric heat flux corrected by the radiated heat flux (note that the conductive heat flux is neglected because measurements are performed in the thermal steady state regime). A typical uncertainty analysis shows that the relative errors on $R a^{*} x$ and $N u_{x}$ are of the order of $\pm 5 \%$. 


\section{http://dx.doi.org/10.21611/qirt.1992.012}

\section{Results and discussions}

\subsection{Introduction}

In this section, some experimental results are presented for different configurations : the vertical flat plate used as a test case, the inclined flat plate (hot surface facing upward and downward) and finally the vertical flat plate with transverse roughness elements. All these configurations were tested in the laminar flow regime.

\subsection{Vertical flat plate}

The vertical flat plate test case, for which theoretical and experimental correlations are available, allows us to check the validity of the experimental procedure. A large number of tests performed with different test conditions give the following experimental correlation :

$$
N u_{x}=0.52\left(R a_{x}^{*}\right)^{0.2} \quad \text { for } \quad 10^{4} \leq R a_{x}^{*} \leq 10^{10}
$$

which is in excellent agreement with previous experimental and theoretical correlations (the largest difference between (5) and existing correlations is only $5 \%$ which is of the order of our experimental uncertainty range).

These first encouraging results allowed us to study the laminar free convection over inclined plates and more complex geometrical configurations.

\subsection{Inclined flat plate}

\subsubsection{Hot surface facing downward}

For this configuration, the same experimental procedure was repeated for several deflection angles and gives the following relation:

$$
N u_{x}=0.52\left(R a^{*}{ }_{x} \cos \alpha\right)^{0.2}
$$

valid for deflection angles up to $55^{\circ}$ with respect to the vertical direction.

This correlation is in fact the extension of equation (5) taking into account the inclination of the plate and can also be presented in terms of average Nusselt number defined as:

$$
\overline{N u}=5 / 4 N u_{X=L}=0.65\left(R a^{*}{ }_{x=L} \cos \alpha\right)^{0.2}
$$

CHEN et al [5] obtained, using a numerical approach, the same correlation (with a constant of proportionality equal to 0.649 ). Our experimental correlation can also be presented in terms of the Grashof number using the definition of the modified Rayleigh number :

$$
\begin{aligned}
N u_{x} & =0.44\left(G r_{x} \operatorname{Pr} \cos \alpha\right)^{0.25} \\
\text { or } \quad \overline{N u} & =0.55\left(G r_{x=L} \operatorname{Pr} \cos \alpha\right)^{0.25}
\end{aligned}
$$

FUJII-IMURA [7] proposed nearly the same correlation as equation (9) but with a constant of proportionality equal to 0.56 . The present correlation (equation (5)) is presented in figure 5 with some existing theoretical or experimental correlations and shows the validity (within the uncertainty range) of our results.

\subsubsection{Hot surface facing upward}

In this case, the angle $\alpha$ was changed from $0^{\circ}$ to $60^{\circ}$ and gives the following correlation :

$$
N u_{x}=0.53\left(R a^{*}{ }_{x} \cos \alpha\right)^{0.2}
$$

This correlation, equivalent to equation (6) within the uncertainty range, shows that the orientation of the heated surface (upward or downward) does not change the heat transfer 
characteristics (assuming that we have a boundary layer type of flow, i.e. for $0^{\circ} \leq \alpha \leq 60^{\circ}$ ), the only significant parameter being the deflection angle $\alpha$.

Nevertheless, for deflection angles larger than $60^{\circ}-70^{\circ}$, a change in the expression of the non dimensional heat transfer law was observed; for example for $\alpha=70^{\circ}$, we obtained:

$$
N u_{x}=0.36\left(R a^{*}{ }_{x} \cos \alpha\right)^{0.23}
$$

with an exponent largely different from the expected value of 0.2 . This change in the thermal behaviour was suspected to be due to the large value of the deflection angle (in this case, the plate is only inclined at $20^{\circ}$ with respect to the horizontal direction) and we can predict a gradual change in the boundary layer state, from a 2D boundary layer (in case of vertical or slightly inclined plates) to the development of a thermal plume for which the boundary layer equations are no longer valid. Examples of flow visualization over the vertical and $70^{\circ}$ inclined plate are presented in figure 6 showing the change in the boundary layer state. This change in the dynamic behaviour could explain the change in the thermal behaviour.

\subsection{Vertical flat plate with roughness elements}

The last geometry analysed in the scope of this study was to determine the effect of disturbing elements located in the laminar boundary layer in order to try to increase the convective heat transfer coefficient. These roughness elements were introduced by means of repeated squared cross section ribs ( $5 \mathrm{~mm}$ by $5 \mathrm{~mm}$ ) oriented perpendicularly to the main flow direction. They were made of high thermal conductive material and fixed in contact with the plate surface. Figure 7 presents the heat transfer coefficient distribution along the $x$-axis obtained with and without roughness elements and shows an increase of the order of $10 \%$ on the heat transfer coefficient in case of disturbing elements. Nevertheless, this $10 \%$ increase is obtained by increasing the exchange surface by $15 \%$. The resulting effect of this geometry is then a small decrease of the global heat transfer performance. The effect of conductive ribs was also experimentally investigated by BHAVNANI and BERGLES [8] who concluded that the performance of ribbed surfaces was below that of a plain plate of equal projected area. According to these authors, an increase of heat transfer can be obtained with stepped surfaces.

Figures $A$ and $B^{*}$ present thermograms obtained respectively on the clean flat plate and on the plate with roughness elements. From figure $B$, we can observe, between two consecutive ribs, small temperature fluctuations in the flow direction generating heat transfer coefficient fluctuations which can be attributed to the alternance of regions of low and high velocities generating boundary layer thickness variations. The presence of separated flow regions (just upstream and downstream of the rib) also induces a decrease of the heat transfer coefficient. In the central region between two consecutive ribs, the flow reattaches again and thus induces an increase of the heat transfer coefficient comparatively to the separated flow regions.

\section{Conclusion}

This study of laminar free convection, actually conducted in our department, has enabled us to set up a satisfactory experimental procedure giving a small uncertainty as well as a good agreement with theoretical and previous experimental correlations. The next step of this study will be focussed on the determination of the geometric configuration giving rise to the best heat transfer performances. This kind of study could also be done in the forced convection regime.

\section{REFERENCES}

[1] SPARROW (E.M.) and GREGG (J.L.) : Laminar free convection from a vertical plate with uniform surface heat flux. Trans. ASME February 1956, p. 435-440.

[2] LEONTIEF (A.) : Théorie des échanges de chaleur et de masse. Editions Mir Moscow, 1985.

[3] SPARROW (E.M.) : Laminar free convection on a vertical plate with prescribed non-uniform wall heat flux or prescribed non-uniform wall temperature. NACA TN 3508, 1955.

[4] DOTSON (J.P.) : Heat transfer from a vertical plate by free convection. MS Thesis, Purdue University, 1954.

* The colour plates of this article 12 are located on page ll of the colour gathering. at the end of the book 
[5] CHEN (T.S.), TIEN (H.C.) and ARMALY (B.F.) : Natural convection on horizontal, inclined and vertical plates with variable surface temperature or heat flux. Int. J. Heat Mass Transfer, Vol. 29, 1986 , p. 1465-1478.

[6] VERMEULEN (J.P.) and BAUDOIN (B.) : Etude de la convection naturelle sur une plaque plane chauffée à flux constant. Société Française des Thermiciens 91 Belfort, May1991.

[7] FUJII (T.) and IMURA (H.) : Natural convection heat transfer across vertical and inclined air layers. J. of Heat Transfer 104 C, 1988, p. 69-102.

[8] BHAVNANI (S.H.) AND BERGLES (A.E.) : Effect of surface geometry and orientation on laminar natural convection heat transfer from a vertical flat plate with transverse roughness elements. Int. J. Heat Mass Transfer, Vol. 33, 1990, p. 965-981.

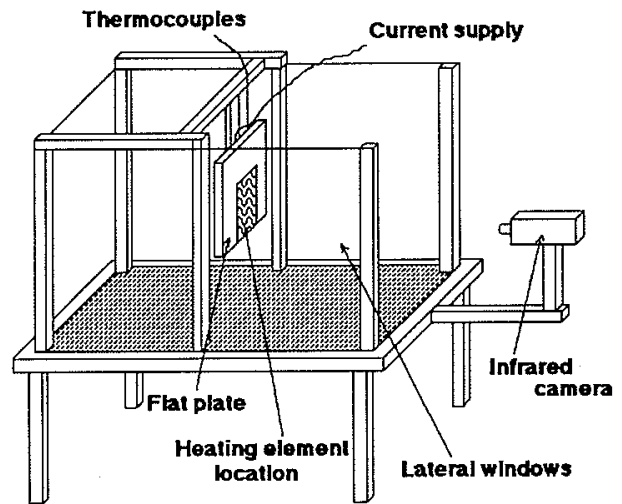

Fig. 1. - Experimental setup

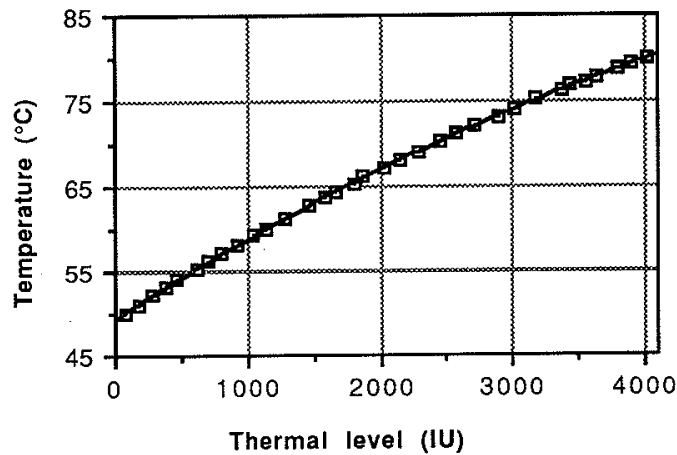

Fig. 3. - Calibration curve

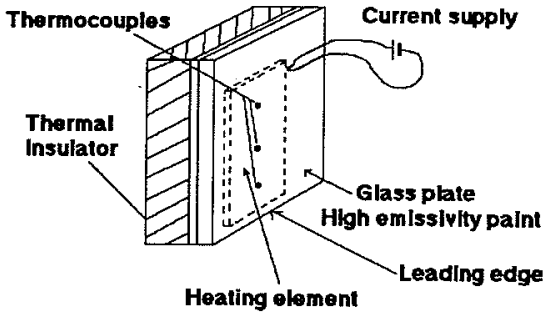

Fig. 2. - Flat plate

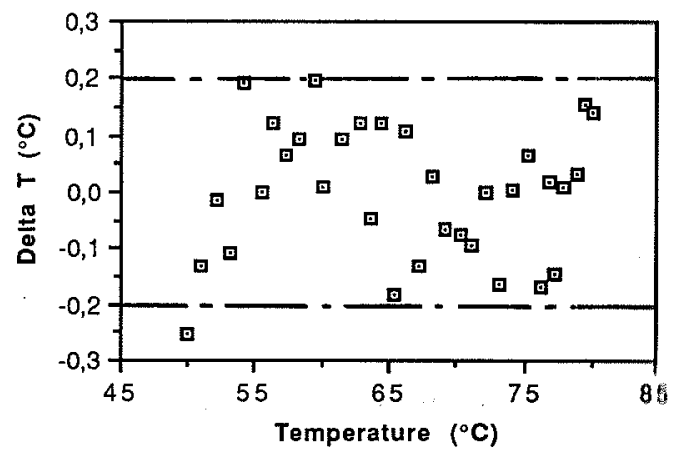

Fig. 4. - Uncertainty range 
http://dx.doi.org/10.21611/qirt.1992.012

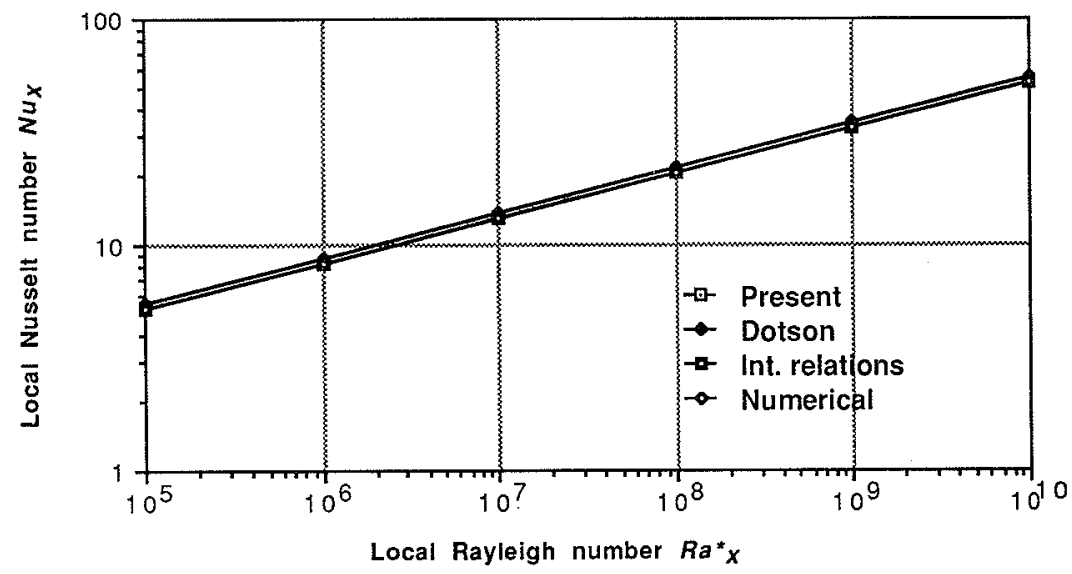

Fig. 5. - Present results and comparison with existing correlations

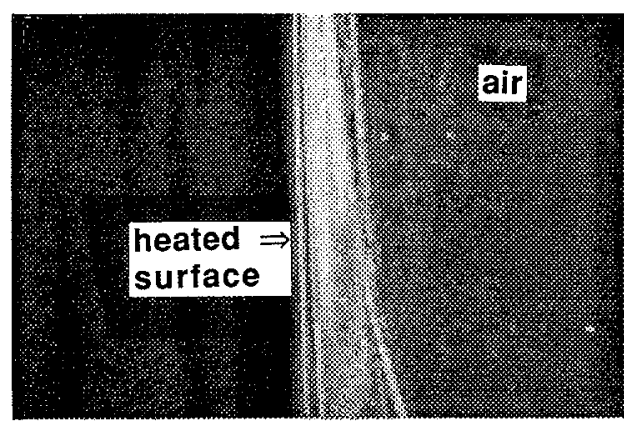

(a) Vertical plate

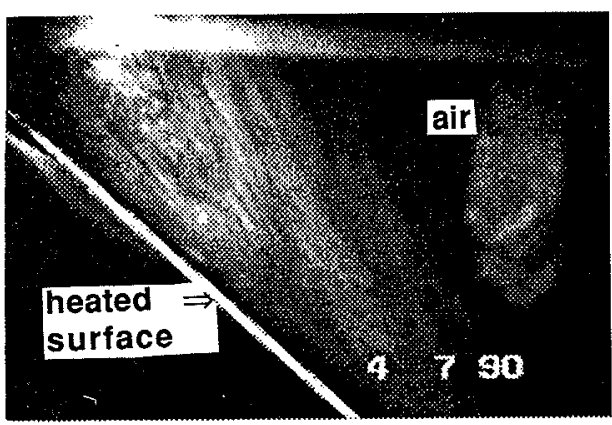

(b) $70^{\circ}$ inclined plate

Fig. 6. - Flow visualizations

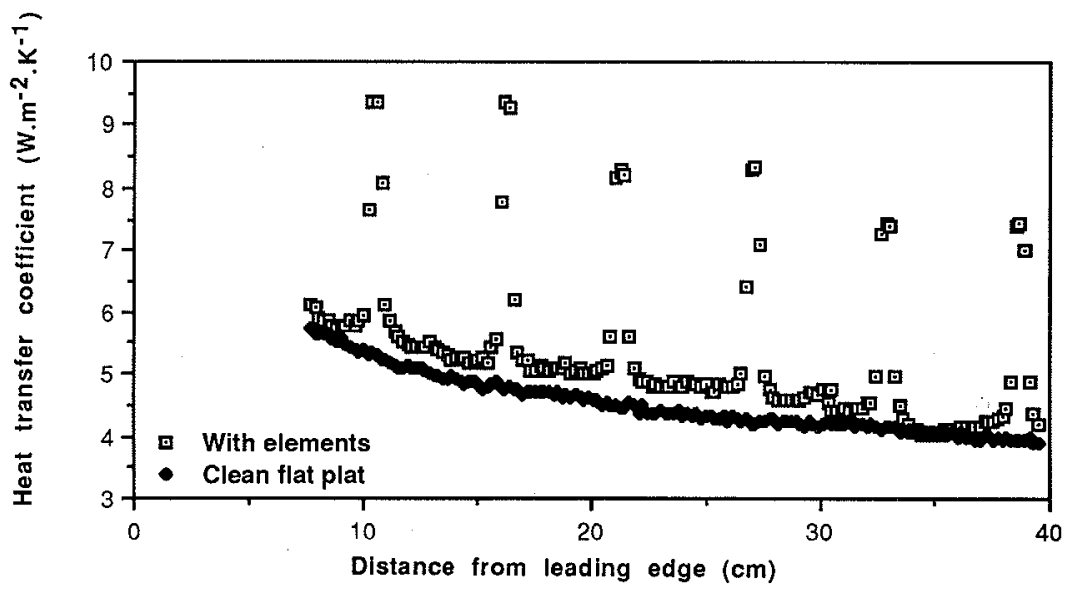

Fig. 7. - Heat transfer coefficient distribution 